\title{
Uso de Aminoácido Exógeno na Prevenção de Injúrias Causadas POR GlyphOSATE NA SOJA RR
}

\author{
Use of Exogenous Amino Acid to Prevent Glyphosate Injury in Glyphosate-Resistant Soybean
}

\author{
ZOBIOLE, L.H.S. ${ }^{2}$, OLIVEIRA JR., R.S. ${ }^{3}$, CONSTANTIN, J. ${ }^{3}$, BIFFE, D.F. ${ }^{2}$ e KREMER, R.J. ${ }^{4}$
}

\begin{abstract}
RESUMO - O aumento da área cultivada de soja resistente ao glyphosate (RR) no Brasil é resultado do beneficio dessa tecnologia no manejo de plantas daninhas. No entanto, a expansão da área de soja RR aumentou significativamente o uso de glyphosate e consequentemente, em alguns casos, têm sido observados sintomas de injúrias na soja RR conhecidos como yellow flashing ou amarelecimento das folhas superiores. Nesse sentido, dois experimentos, em diferentes anos, foram conduzidos em delineamento inteiramente casualizado, com quatro repetições. O primeiro teve o objetivo de avaliar a influência do glyphosate na soja RR nas variáveis fotossintéticas, nos parâmetros de nodulação e na biomassa seca da parte aérea e raiz, realizando-se a comparação entre os tratamentos BRS 242 RR sem glyphosate, BRS 242 RR + glyphosate e a isolinha parental não-RR cv. Embrapa 58, submetidas a uma dose de glyphosate de 1.200 g e.a. ha-1, aplicada no estádio V4. O segundo experimento foi conduzido visando reavaliar as variáveis fotossintéticas, de produção de biomassa e nodulação afetadas na soja RR pelo glyphosate no primeiro experimento. Entretanto, no segundo experimento foi avaliada a utilização de diversas modalidades de aplicação de aminoácidos (a.a.), sendo os diferentes tratamentos (sem a.a.; tratamento de semente com a.a; tratamento de semente com a.a. + aplicação foliar de a.a.; sem tratamento de sementes com a.a; e com aplicação foliar de a.a) combinados com diferentes doses de glyphosate (1.200 e $2.400 \mathrm{ge.a}$. ha ${ }^{-1}$ ), objetivando uma provável recuperação das plantas de soja com sua utilização exógena. Em ambos os experimentos, as variáveis fotossintéticas, os parâmetros de nodulação e biomassa seca da parte aérea e raiz foram afetados pela aplicação do glyphosate, porém o segundo experimento evidenciou, de modo geral, que o uso de aminoácidos via tratamento de sementes associado com aplicação foliar pode ser uma estratégia para prevenir os efeitos indesejáveis desse herbicida na cultura da soja RR.
\end{abstract}

Palavras-chave: fotossíntese, biomassa, nodulação, herbicida, Rhizobium.

\begin{abstract}
Cultivation of glyphosate-resistant (GR) soybeans has increased in Brazil as a result of the application of this technology in weed management systems developed for this crop. However, the expansion of GR soybean production has significantly increased the use of glyphosate and, in some cases, resulted in injury symptoms observed in GR soybean, known as "yellow flashing" or yellowing of the upper leaves. Thus, two experiments were conducted in different years. The first experiment aimed to evaluate the influence of glyphosate on GR soybeans regarding the photosynthetic variables, nodule parameters, and shoot and root dry biomass by comparing cultivar BRS 242 GR without glyphosate and BRS $242 R R+$ glyphosate at $1.200 \mathrm{~g} \mathrm{ha}^{-1}$ at V4 growth stage, to the near isogenic non-GR parental line cv. Embrapa 58. The second experiment aimed to reassess the same parameters in GR soybeans at the V4 stage treated with glyphosate, plus the application of various amino acids, to evaluate the expected recovery of soybean growth under the exogenous use of supplemental amino acids. In general, the photosynthetic variables, nodulation parameters and shoot and root dry biomass were affected by glyphosate; however, the use of amino acids may be a strategy to prevent the undesirable effects of this herbicide on GR soybean
\end{abstract}

Keywords: photosynthesis, biomass, nodulation, herbicide, Rhizobium

Recebido para publicação em 16.12.2009 e na forma revisada em 3.9.2010.

2 Doutorando do curso do Programa de Pós-Graduação em Agronomia, Universidade Estadual de Maringá -UEM, Av. Colombo, 5790, Bloco J45, 87020-900 Maringá-PR, <lhzobiole@uol.com.br>; ${ }^{3}$ Professor Associado, Dep. de Agronomia, UEM; ${ }^{4}$ United States Department of Agriculture, Agricultural Research Service, Cropping Systems \& Water Quality Research Unit, University of Missouri, 327 Anheuser-Busch Natural Resources Building, 65201, Columbia, MO, USA.

Planta Daninha, Viçosa-MG, v. 28, n. 3, p. 643-653, 2010 


\section{INTRODUÇÃO}

No Brasil, a área cultivada com soja resistente ao glyphosate (RR) deve alcançar na safra $2009 / 2010$ cerca de $65 \%$ da área total cultivada de soja, e continua aumentando significativamente. A cada ano, empresas de biotecnologia investem bilhões de dólares para o desenvolvimento de variedades resistentes a herbicidas. No entanto, com o aumento do uso da tecnologia da soja RR, muitos agricultores têm notado que alguns cultivares RR apresentam injúrias visuais logo após a aplicação do glyphosate em pós-emergência (Santos et al., 2007; Zablotowicz \& Reddy, 2007).

Observações em campo no Brasil e na região centro-norte dos Estados Unidos levaram a relatos de deficiências de $\mathrm{Fe}, \mathrm{Zn}$ e $\mathrm{Mn}$ na soja RR após a utilização frequente de glyphosate (Huber, 2006; Johal \& Huber, 2009). O sintoma típico observado em campo após a aplicação do glyphosate é conhecido como yellow flashing ou amarelecimento das folhas superiores. Recentes pesquisas demonstraram que esse sintoma clorótico nas culturas RR pode ser atribuído à imobilização de cátions bivalentes, como Fe e Mn (Huber, 2006; Bott et al., 2008; Zobiole et al., 2010a). Uma possibilidade para ocorrer a menor disponibilidade desses cátions é que o glyphosate, por ser um ácido fosfônico e "quelador" de cátions metálicos (Kabachnik et al., 1974), poderia formar quelatos com cátions bivalentes e trivalentes. Provavelmente, a duração desse amarelecimento depende da habilidade da planta de se recuperar pela absorção radicular dos elementos que foram imobilizados pelo glyphosate nos tecidos foliares (Cakmak et al., 2009).

De acordo com Reddy et al. (2004), o sintoma clorótico indesejável na soja RR tem sido atribuído ao acúmulo do primeiro metabólito fitotóxico do glyphosate, conhecido como AMPA (ácido aminometilfosfônico), o qual é um dos responsáveis pela diminuição da biomassa seca da parte aérea e raiz e do teor de clorofila (Reddy et al., 2000; King et al., 2001; Zablotowicz \& Reddy, 2004). Esses sintomas não têm ocorrido apenas em solos com baixa disponibilidade natural de micronutrientes, como os dos cerrados, mas também em áreas com adequada ou até alta fertilidade, como os Latossolos Vermelhos Eutroférricos, distribuídos pelas regiões norte e oeste do Paraná. A ocorrência desse amarelecimento tem levado muitos produtores ao uso intensivo de fertilizantes foliares como alternativa para amenizar ou suprir essa provável deficiência nutricional, sem, no entanto, haver necessariamente a recuperação da cultura.

Sabe-se que bioestimulantes são utilizados para aumentar o crescimento e a produtividade da soja, sob o argumento de que esses produtos podem aumentar a atividade microbiológica, biodisponibilidade de nutrientes e mineralização da matéria orgânica (Subler et al., 1998; Chen et al., 2002). Contudo, até o momento não existem estudos específicos visando avaliar a aplicação de bioestimulantes na recuperação dos efeitos indesejáveis do glyphosate na soja RR.

Existem pesquisas que relatam que o suprimento exógeno de aminoácidos (a.a.) pode reduzir a inibição do crescimento em plantas atingidas por herbicidas que agem no metabolismo de aminoácidos (Jaworski, 1972; Roisch \& Lingens, 1974; Haderlie et al., 1977; Gresshoff, 1979). Nas décadas de 1980 e 1990, estudos visando descobrir os mecanismos de ação dos herbicidas eram feitos a partir da aplicação de determinadas moléculas em plantas, incubando-as posteriormente com diversas combinações de aminoácidos. Dessa forma, pela adição de aminoácido no meio de cultura, e com a recuperação dos sintomas, era possivel descobrir qual o sítio de ação do herbicida. Ray (1984) demonstrou que a adição de valina e isoleucina a herbicidas inibidores da acetolactato sintase (ALS), como o chlorsulfuron, reduziu completamente a inibição do crescimento de raízes de ervilha (Pisum sativum). De forma semelhante, a adição desses mesmos aminoácidos reverteu a inibição do crescimento de milho, causada pelo herbicida imazapir, em trabalho conduzido por Shaner et al. (1984).

Em relação aos herbicidas inibidores da enzima 5-enol-piruvil-3-shikimato-fosfato sintetase - EPSPs, um dos primeiros trabalhos conduzidos visando suprimir os sintomas do herbicida glyphosate pela adição da mistura de fenilalanina, tirosina e triptofano foi descrito por Jaworski (1972). Nesse trabalho, a utilização desses três aminoácidos preveniu a 
inibição do crescimento de Lemma gibba e da bactéria Rhizobium japonicum. Esses mesmos resultados foram confirmados por Santos et al. (2005), os quais descreveram que a adição desses aminoácidos aromáticos ao meio de cultura com $B$. japonicum preveniu a inibição do crescimento pelo glyphosate, pelo fato de esse microrganismo possuir esta enzima sensivel ao glyphosate (Moorman et al., 1992; De Maria et al., 2006). Forlani et al. (1997) também relatam que a adição de aminoácidos aromáticos preveniu parcialmente a inibição do crescimento de raízes de pepino (Cucumis sativus) causada pelo glyphosate e pelo ácido $\mathrm{N}$-pirimidilaminometilenobifosfônico (Forlani et al., 1997).

Considerando que os problemas causados pelo herbicida glyphosate na cultura da soja RR em todo o mundo são um tema bastante atual e que há um apenas um limitado conhecimento no Brasil a respeito da resposta de cultivares de soja RR à aplicação de glyphosate, o primeiro experimento deste trabalho foi instalado com o objetivo de avaliar a influência do glyphosate na soja RR, e o segundo, de reavaliar os efeitos indesejáveis do glyphosate na soja $R R$ e também a utilização de diversas modalidades de aplicação de aminoácidos visando uma provável recuperação das plantas de soja.

\section{MATERIAL E MÉTODOS}

Dois experimentos foram conduzidos em casa de vegetação (25-35: 20-22 ${ }^{\circ} \mathrm{C}$ dia/noite), sob condições naturais de luminosidade, na Universidade Estadual de Maringá. O primeiro foi conduzido entre outubro de 2007 e fevereiro de 2008; e o segundo, entre dezembro de 2008 e fevereiro de 2009 , a $23^{\circ} 25^{\prime}$ de latitude sul e $51^{\circ} 57^{\prime}$ de longitude oeste de Greenwich, numa altitude de $542 \mathrm{~m}$.

As unidades experimentais foram constituídas de vasos de polietileno de $5,0 \mathrm{dm}^{3}$, preenchidos com solo argiloso (Latossolo Vermelho nitossólico), proveniente do horizonte A coletado no Centro Tecnológico de Irrigação da Universidade Estadual de Maringá (MaringáPR). Após coleta, o solo foi seco ao ar e passado em peneira com malha 10 mesh. Os resultados das análises químicas e granulométrica dos dois solos dos experimentos encontram-se na Tabela 1. As propriedades físicoquímicas foram determinadas de acordo com procedimentos estabelecidos pela Embrapa (1997).

Ambos os experimentos foram conduzidos em delineamento inteiramente casualizado, com quatro repetições. No primeiro, foram avaliados os tratamentos BRS 242 RR sem glyphosate, BRS $242 \mathrm{RR}+$ glyphosate e a isolinha parental não-RR cv. Embrapa 58, submetidos a uma dose de glyphosate de 1.200 g e.a. ha-1 aplicada no estádio V4. O segundo experimento foi conduzido em esquema fatorial $(3 \times 4)+1$, sendo o primeiro fator representado pelas doses de glyphosate $(0,1.200 \mathrm{e}$ $2.400 \mathrm{~g}$ a.e. $\left.\mathrm{ha}^{-1}\right)$ e o segundo pelas diversas modalidades de aplicação de aminoácido (sem fornecimento de aminoácidos - SEM AA; com fornecimento de aminoácidos via tratamento de sementes - TRATSEM; com fornecimento de aminoácidos via tratamento de sementes e via foliar em mistura com glyphosate TRATSEM + FOL; e apenas com fornecimento de aminoácidos via foliar em mistura com glyphosate - FOL). O tratamento adicional $(+1)$ foi constituído pela isolinha parental não-RR cv. Embrapa 58 e não recebeu nenhuma aplicação de glyphosate nem de aminoácido.

Tabela 1 - Análise química e granulométrica da amostra de solo utilizada nos experimentos. Maringá-PR, 2007 e 2008

\begin{tabular}{|c|c|c|c|c|c|c|c|c|c|c|c|c|c|}
\hline \multicolumn{2}{|c|}{$\mathrm{pH}$} & $\mathrm{C}$ & $\mathrm{P}$ & $\mathrm{Al}^{+++}$ & $\mathrm{H}^{+}+\mathrm{Al}^{+++}$ & $\mathrm{Ca}^{++}+\mathrm{Mg}^{++}$ & $\mathrm{Ca}^{++}$ & $\mathrm{K}^{+}$ & $\mathrm{S}$ & $\mathrm{Fe}^{++}$ & $\mathrm{Zn}^{++}$ & $\mathrm{Cu}^{++}$ & $\mathrm{Mn}^{++}$ \\
\hline$\left(\mathrm{CaCl}_{2}\right)$ & $\left(\mathrm{H}_{2} \mathrm{O}\right)$ & $\left(\mathrm{g} \mathrm{dm}^{-3}\right)$ & $\left(\mathrm{mg} \mathrm{dm}^{-3}\right)$ & \multicolumn{6}{|c|}{$\left(\mathrm{cmol}_{\mathrm{c}} \mathrm{dm}^{-3}\right)$} & \multicolumn{4}{|c|}{$\left(\mathrm{mg} \mathrm{dm}^{-3}\right)$} \\
\hline 5,70 & 6,10 & 25,82 & 18,0 & 0,0 & 3,97 & 11,93 & 8,71 & 1,13 & 9,61 & 64,43 & 13,87 & 25,61 & 148,58 \\
\hline \multicolumn{4}{|c|}{ Areia grossa } & \multicolumn{3}{|c|}{ Areia fina } & \multicolumn{3}{|c|}{ Silte } & & \multicolumn{3}{|c|}{ Argila } \\
\hline \multicolumn{14}{|c|}{$\left(\mathrm{g} \mathrm{kg}^{-1}\right)$} \\
\hline \multicolumn{3}{|c|}{40} & & \multicolumn{3}{|c|}{120} & \multicolumn{3}{|c|}{90} & & \multicolumn{3}{|c|}{750} \\
\hline
\end{tabular}


Antes da semeadura, as sementes de soja (Glycine $\max$ ) cv. BRS $242 \mathrm{RR}$ e sua isolinha parental não-RR cv. Embrapa 58 foram tratadas com $200 \mathrm{~mL} 100 \mathrm{~kg}^{-1}$ de sementes com a mistura de $200 \mathrm{~g} \mathrm{~L}^{-1}$ carboxim $+200 \mathrm{~g} \mathrm{~L}^{-1} \mathrm{de}$ thiram, $13,5 \mathrm{~g} \mathrm{~L}^{-1}$ de cobalto e $135 \mathrm{~g} \mathrm{~L}^{-1} \mathrm{de}$ molibdênio. As sementes foram então inoculadas com $300 \mathrm{~mL} 100 \mathrm{~kg}^{-1}$ de sementes da cultura de Bradyrhizobium elkanii, estirpes SEMIA 587 e SEMIA 5019, na concentração de $5 \times 10^{9}$ rizóbios por grama. Cinco sementes foram semeadas por vaso na profundidade de $3 \mathrm{~cm}$. Quando as plantas atingiram o estádio $\mathrm{V} 1$, foi realizado o desbaste, deixando-se apenas uma planta por vaso

Plantas no estádio V5 (35 dias após semeadura - DAS) foram sujeitas à aplicação de glyphosate formulado comercialmente como sal de isopropilamina (480 g e.a. $\mathrm{L}^{-1}$ ). As aplicações foram feitas com os vasos colocados do lado de fora da casa de vegetação, utilizando-se barra equipada com quatro bicos com pontas tipo leque (Teejet XR 110.02), espaçados de $50 \mathrm{~cm}$ entre si, posicionados na altura de $50 \mathrm{~cm}$ das plantas, com volume relativo de calda de $190 \mathrm{~L} \mathrm{ha}^{-1}$. As condições climáticas no momento da aplicação eram de temperatura entre 24 e $28{ }^{\circ} \mathrm{C}$, umidade relativa do ar entre 80 e $90 \%$, solo úmido e velocidade do vento entre $6 \mathrm{e}$ $8 \mathrm{~km} \mathrm{~h}^{-1}$. Após as aplicações do herbicida, os vasos retornaram à casa de vegetação e foram irrigados apenas no dia seguinte, garantindo assim a absorção foliar do herbicida. Os vasos foram irrigados diariamente, para conservar o solo úmido, e mantidos livres de plantas daninhas por meio de capinas manuais.

Os tratamentos com aminoácidos consistiram em tratamento de sementes na dose de $5 \mathrm{~mL} \mathrm{~kg}$ sementes $^{-1}$ ou na aplicação conjunta com glyphosate na dose de $2 \mathrm{~L}$ ha ${ }^{1}$ do produto comercial AminoPlus ${ }^{\circledR}$, Ajinomoto, São Paulo, Brasil. Este produto apresenta a seguinte composição: alanina $(1,164 \%)$, arginina (0,189\%), ácido aspártico (1,943\%), ácido glutâmico $(3,316 \%)$, glicina $(0,202 \%)$, isoleucina $(0,171 \%)$, leucina $(0.268 \%)$, lisina $(0,240 \%)$, fenilalanina $(0,143 \%)$, serina $(0,179 \%)$, treonina $(0,188 \%)$, triptofano $(0,175 \%)$, tirosina $(0,122 \%)$, valina $(0,288 \%)$ e os nutrientes: $\mathrm{N}-11 \%$ e $\mathrm{K}_{2} \mathrm{O}-1 \%$.

Quando as plantas atingiram o estádio R1 (aos 46 e 48 DAS para o primeiro e segundo experimentos, respectivamente), foram feitas avaliações da taxa fotossintética ( $\mu \mathrm{mol}$ $\mathrm{CO}_{2} \mathrm{~m}^{-2} \mathbf{s}^{-1}$ ) em um dia sem nebulosidade com fluxo de fótons fotossinteticamente ativos (FFFA) de $\pm 1.500 \mu \mathrm{mol} \mathrm{m} \mathrm{m}^{-2} \mathrm{~s}^{-1}$ PARi, com um sistema fechado portátil de fotossintese, utilizando o equipamento ADC model LCpro+(Infra Red Gas Analyser, Analytical Development Co. Ltd, Hoddesdon, UK). O teor de clorofila (unidades SPAD) foi avaliado no equipamento Minolta (SPAD502 meter).

As avaliações foram realizadas entre $9 \mathrm{~h}$ e $12 \mathrm{~h}$, tomando-se as medidas na parte mediana do segundo trifólio completamente expandido (folha diagnóstica). Após essas avaliações, as partes aéreas das plantas foram cortadas rente ao solo, embaladas em sacos de papel e secas em estufa de circulação forçada de ar a 65 $70{ }^{\circ} \mathrm{C}$ até peso constante. As raízes foram lavadas em água corrente e, em seguida, procedeu-se ao destaque e contagem do número de nódulos, para posterior secagem do material e determinação da sua matéria seca. No entanto, no segundo experimento, imediatamente antes (V4, 30 DAS) e após (V7, 42 DAS) aplicações dos diferentes tratamentos foram avaliadas as taxas fotossintéticas e o indice SPAD. Da mesma forma, no segundo experimento, as demais variáveis, como massa seca da parte aérea, raiz, nódulos, número de nódulos e altura, foram avaliadas no estádio R1.

Os erros dos dados passaram pelos testes de Levene e Shapiro-Wilk, com o objetivo de avaliar a sua variância e normalidade. Os dados de ambos os experimentos foram comparados pelo teste de agrupamento de Scott Knott a 5\% de probabilidade, utilizando-se o software SISVAR (Ferreira, 1999), e também comparados com a isolinha parental não-RR pelo teste de Dunnet a 5\% probabilidade pelo PROC GLM (SAS, 2001).

\section{RESULTADOS E DISCUSSÃO}

No primeiro experimento no estádio $R 1$ (46 DAS), não houve diferença no índice SPAD, porém a taxa fotossintética (A) foi diminuída com a aplicação de glyphosate no cultivar BRS 242 RR (Tabela 2), quando comparado com o mesmo cultivar sem herbicida ou com sua 
Tabela 2 - Taxa fotossintética (A), SPAD, biomassa seca da parte aérea, da raiz e de nódulos e número de nódulos por planta avaliados no estádio R1 (experimento 1)

\begin{tabular}{|l|c|c|c|c|c|c|}
\hline \multirow{2}{*}{ Tratamento } & $\mathrm{A}$ & SPAD & Parte aérea & Raiz & Nódulos & Nódulos \\
\cline { 2 - 7 } & $\left(\mu \mathrm{mol} \mathrm{CO}_{2} \mathrm{~m}^{-2} \mathrm{~s}^{-1}\right)$ & \multicolumn{2}{|c|}{ (unidade) } & \multicolumn{2}{|c|}{ (g por planta) } & (unidade por planta) \\
\hline BRS 242 GR & $16,49 \mathrm{a}$ & $35,7 \mathrm{a}$ & $12,62 \mathrm{a}$ & $7,24 \mathrm{a}$ & $1,72 \mathrm{a}$ & $239,87 \mathrm{a}$ \\
\hline BRS 242 GR +glyphosate & $14,42 \mathrm{~b}$ & $31,5 \mathrm{a}$ & $9,62 \mathrm{~b}$ & $5,08 \mathrm{~b}$ & $1,13 \mathrm{~b}$ & $148,33 \mathrm{~b}$ \\
\hline Isolinha parental (Embrapa 48) & $20,97 \mathrm{a}$ & $37,3 \mathrm{a}$ & $13,54 \mathrm{a}$ & $4,48 \mathrm{~b}$ & $0,92 \mathrm{~b}$ & $156,92 \mathrm{~b}$ \\
\hline \multicolumn{1}{|c|}{ CV(\%) } & 24,26 & 23,68 & 20,49 & 24,91 & 21,69 & 28,80 \\
\hline
\end{tabular}

Médias seguidas pela mesma letra minúscula na coluna não apresentam diferenças estatísticas significativas pelo teste LSD a $5 \%$ de probabilidade.

isolinha parental não-RR (Embrapa 58). Isso indica que, mesmo na ausência de sintomas perceptíveis de clorose após a aplicação desse herbicida na soja RR, ainda assim é possível que haja efeitos de fitointoxicação capazes de afetar, mesmo que de forma transitória, a intensidade de fixação de carbono pelas plantas. Estudos anteriores demonstraram que a taxa fotossintética (A) e o indice SPAD foram severamente reduzidos pelo glyphosate em todos os grupos de maturação de cultivares de soja RR avaliados em diferentes solos, porém não houve diferença entre os tratamentos sem glyphosate na soja RR e suas respectivas isolinhas parentais (Zobiole et al., 2010a).

No presente trabalho, plantas tratadas com glyphosate exibiram sintomas leves de clorose, os quais devem estar relacionados com a diminuição da taxa fotossintética, devido ao dano do glyphosate à clorofila (Kitchen et al., 1981a; Lee, 1981; Reddy et al., 2004) ou à imobilização de $\mathrm{Mg}$ e $\mathrm{Mn}$ requeridos para a formação de clorofila e para a fotossintese, respectivamente (Beale, 1978; Taiz \& Zeiger, 1998). O principal metabólito do glyphosate em plantas é o ácido aminometilfosfônico (AMPA), o qual pode também estar associado às injúrias na soja RR tratada com glyphosate (Pline et al., 1999; Reddy et al., 2001; Duke et al., 2003; Reddy et al., 2004).

Ainda no primeiro experimento, o número de nódulos e a biomassa seca da parte aérea, da raiz e dos nódulos também foram severamente reduzidos pelo glyphosate (Tabela 2). A diminuição da nodulação causada pelo glyphosate pode ocorrer devido ao fato de que o microrganismo fixador de nitrogênio, Bradyrhizobium japonicum, possui a enzima EPSPs sensivel ao glyphosate, podendo ocorrer, dessa forma, o acúmulo dos ácidos chiquímico, hidroxibenzoico e protocatecúico e, consequentemente, a inibição da sintese de aminoácidos aromáticos, induzindo assim a morte do simbionte (Moorman et al., 1992; Bellalloui et al., 2006; De Maria et al., 2006).

A biomassa seca de nódulos é um dos principais parâmetros para quantificar a eficiência de fixação biológica de nitrogênio em soja, e um coeficiente de variação $(\mathrm{CV})$ aceitável deve ser < 33\% (Souza et al., 2008a,b). No presente estudo, o CV para biomassa seca de nódulos foi de $21,69 \%$. Para o número de nódulos, o CV de $28,80 \%$ é classificado como médio, uma vez que essa variável é considerada de alta variação (Souza et al., 2008a); portanto, os CVs para os parâmetros de nodulação estão dentro de uma faixa aceitável, o que induz a considerar que o glyphosate interferiu efetivamente na nodulação da soja BRS 242 RR. Esses resultados estão de acordo com os de outros autores, os quais também observaram redução na nodulação pelo uso do glyphosate (King et al., 2001; Reddy \& Zablotowicz, 2003; Oliveira et al., 2008; Zobiole et al., 2010b). No entanto, a isolinha parental apresentou menor biomassa de raiz e de nódulos em relação à soja $R R$ sem aplicação do glyphosate, provavelmente pela característica do cultivar em apresentar menor sistema radicular, contribuindo para o menor suprimento de nutrientes para os nódulos, o que se reflete no menor acúmulo de massa.

Embora os efeitos do glyphosate sobre a soja RR sejam dependentes de fatores como variedade, grupo de maturação, época de aplicação e dose, trabalhos anteriores desenvolvidos por nosso grupo de trabalho já demonstraram o efeito deletério desse herbicida sobre a 
nodulação de um grande número de variedades de soja RR cultivadas no Brasil (Oliveira Jr. et al., 2008).

$\mathrm{O}$ efeito mais pronunciado do glyphosate ocorreu na parte aérea, possivelmente em razão do limitado período de tempo que essa cultivar dispõe para recuperar-se, uma vez que pertence ao grupo de maturação precoce. Efeitos negativos no acúmulo de biomassa ocorrem provavelmente devido aos efeitos aditivos da diminuição da taxa fotossintética (Tabela 2) e da redução na concentração dos nutrientes na parte aérea (Zobiole et al., 2010a). Da mesma forma, diversos autores, utilizando diferentes doses de glyphosate -1.680 g a.e. ha ${ }^{-1}$ em Reddy et al. (2000) e $6.300 \mathrm{~g}$ a.e. ha ${ }^{-1}$ em King et al. (2001) -, encontraram reduções na biomassa da parte aérea e de raízes de soja $R R$. Esses resultados também encontram respaldo nos trabalhos desenvolvidos por Bott et al. (2008), os quais observaram que a aplicação do glyphosate na soja $R R$ reduziu significativamente a biomassa e a elongação da raiz.

No segundo experimento, a avaliação da taxa fotossintética (A) e do índice SPAD, realizada imediatamente antes da aplicação do glyphosate (soja no estádio V4), sugere que a simples aplicação da fonte exógena de aminoácidos via tratamento de sementes não interferiu de forma significativa nesses parâmetros (Tabela 3). Demonstrou-se ainda que não havia diferenças prévias entre os valores observados nas plantas que seriam submetidas às doses de glyphosate e a isolinha parental (Embrapa 48). Imediatamente após a aplicação (soja no estádio V7) da dose de 2.400 g e.a. ha-1, tanto A quanto SPAD foram reduzidos (em relação à dose zero de glyphosate) com ou sem a adição de aminoácidos, mostrando também valores inferiores aos da isolinha parental quando comparados pelo teste de Dunnet a 5\% de probabilidade (Tabela 4). Utilizando a dose recomendada ( $1.200 \mathrm{~g}$ a.e. $\mathrm{ha}^{-1}$ ), a taxa fotossintética foi reduzida apenas na ausência de fonte exógena de aminoácidos ou quando estes foram supridos apenas via tratamento de sementes. O fornecimento de aminoácidos em associação com a aplicação do glyphosate reduziu os efeitos negativos do herbicida sobre a taxa fotossintética da cultura. Em razão do período de tempo entre o tratamento de sementes e a aplicação do glyphosate em pós-emergência, é possível que o potencial de mitigação da solução de aminoácidos fornecida às plantas de soja tenha sido minimizado. Já para a avaliação do teor de clorofila (SPAD), somente o tratamento TRATSEM+FOL foi suficientemente efetivo para evitar os sintomas indesejáveis.

Plantas de soja RR que não receberam a aplicação de glyphosate apresentaram A de $11,02 \mu \mathrm{mol} \mathrm{CO} \mathrm{Cm}^{-2} \mathbf{s}^{-1}$ (Tabela 4). Procópio et al. (2004) encontraram valores similares para A $\left(11-12 \mu \mathrm{mol} \mathrm{CO} \mathrm{Cm}^{-2} \mathbf{s}^{-1}\right)$ aos 39 DAS em Glycine max e Phaseolus vulgaris, sendo considerado um valor ótimo para essa fase vegetativa (Liu et al., 2005). Tratamentos sem glyphosate e com a adição de aminoácidos nas diversas modalidades não apresentaram aumento nos valores dos parâmetros fotossintéticos avaliados, provavelmente por não ter ocorrido o bloqueio da rota do ácido chiquímico, impedindo a conversão de fotoassimilados. Além disso, a adição de carbono (aminoácido) no sistema-planta não leva a aumento de fotossintese e biomassa, pelo fato de aproximadamente $20 \%$ do carbono fixado pelas plantas superiores ser sintetizado em aminoácidos aromáticos, vitaminas, lignina, alcaloides e compostos fenólicos (Kishore \& Shah, 1988). De acordo com o conceito do nivel de suficiência, existem niveis definidos, por exemplo, para os nutrientes individuais no solo. Segundo esse conceito, abaixo desse nível as culturas responderão aos fertilizantes adicionados, e acima, a cultura provavelmente não responderá (Eckert, 1987). É possivel que um conceito semelhante seja válido para a aplicação exógena de aminoácidos.

Os mesmos resultados observados para os tratamentos sem glyphosate e com adição de aminoácidos foram também obtidos na avaliação realizada no estádio $R 1$, não se observando aumento da fotossintese com a utilização dos tratamentos com aminoácidos (Tabela 5). Comparando as diversas modalidades de aplicação de aminoácido na dose de 1.200 g e.a. ha-1 de glyphosate, não houve diferença significativa entre elas em relação a A, porém qualquer forma de fornecimento de aminoácidos preveniu o decréscimo da taxa fotossintética em relação às plantas que não receberam aminoácidos. No entanto, a utilização de TRATSEM + FOL preveniu a 
diminuição da A na maior dose de glyphosate; para o índice SPAD, apenas FOL não mostrou redução no teor de clorofila causada pelo glyphosate.

Biomassas secas da parte aérea e de raízes foram afetadas pela aplicação de glyphosate na ausência de suprimento com aminoácidos, porém não houve diferenças significativas nos efeitos entre as doses de herbicida (Tabela 6). Como esperado, a aplicação de aminoácidos no tratamento sem glyphosate não reverteu em benefícios; contudo, o seu uso nas plantas que receberam a aplicação do glyphosate serviu para prevenir reduções no acúmulo de biomassa das plantas de soja. Comparando-se as modalidades de utilização de aminoácidos para as diferentes doses, a aplicação de TRATSEM foi inferior à de TRATSEM + FOL e FOL na produção de biomassa seca da parte aérea em ambas as doses (Tabela 6). Em relação à produção de biomassa seca de raiz, a modalidade de fornecimento exógeno de aminoácidos que apresentou o melhor efeito foi TRATSEM +
FOL, porém, comparando a biomassa seca total, não houve diferença entre TRATSEM + FOL e FOL; em ambas as modalidades a produção de biomassa e a altura de plantas foram preservadas (Tabelas 6 e 7). Uma das hipóteses para as reduções ocorridas no acúmulo de biomassa seca da parte aérea e da raiz em soja $R R$ na presença do glyphosate, como discutido anteriormente, é o processo de degradação desse herbicida dentro da planta, que resulta na formação do AMPA, conhecida fitotoxina (Duke et al., 2003; Reddy et al., 2004).

No tocante à nodulação, a biomassa seca de nódulos e o número de nódulos foram afetados pelo glyphosate (Tabela 7). O uso de aminoácidos nas modalidades TRATSEM + FOL e FOL levou a uma menor redução de biomassa e número de nódulos, porém, comparando-se entre essas duas modalidades e nas diferentes doses de glyhosate, o melhor tratamento foi TRATSEM + FOL, com consequente menor redução de biomassa seca de nódulos Esses dados estão de acordo com os observados

Tabela 3 - Taxa fotossintética e SPAD no estádio V4, antes da aplicação dos tratamentos com glyphosate e aminoácidos nas plantas de soja RR (experimento 2)

\begin{tabular}{|c|c|c|c|c|c|c|c|c|}
\hline \multirow{2}{*}{$\begin{array}{l}\text { Glyphosate } \\
\left(\mathrm{g} \text { a.e. } \mathrm{ha}^{-1}\right)\end{array}$} & \multicolumn{4}{|c|}{ Taxa fotossintética (A) $\left.\mathrm{mol} \mathrm{CO}_{2} \mathrm{~m}^{-2} \mathrm{~s}^{-1}\right)$} & \multicolumn{4}{|c|}{ SPAD (unidade) } \\
\hline & SEM AA & TRAT SEM & $\begin{array}{l}\text { TRATSEM + } \\
\text { FOL }\end{array}$ & FOL & SEM AA & TRAT SEM & $\begin{array}{l}\text { TRATSEM }+ \\
\text { FOL }\end{array}$ & FOL \\
\hline 0 & $10,62 \mathrm{Aa}$ & $8,16 \mathrm{Aa}$ & $9,24 \mathrm{Aa}$ & $9,97 \mathrm{Aa}$ & $23,57 \mathrm{Aa}$ & $22,71 \mathrm{Aa}$ & $24,28 \mathrm{Aa}$ & $23,71 \mathrm{Aa}$ \\
\hline 1.200 & $9,80 \mathrm{Aa}$ & $9,09 \mathrm{Aa}$ & $9,85 \mathrm{Aa}$ & $10,48 \mathrm{Aa}$ & $22,97 \mathrm{Aa}$ & $24,40 \mathrm{Aa}$ & $25,41 \mathrm{Aa}$ & $22,62 \mathrm{Aa}$ \\
\hline 2.400 & $9,29 \mathrm{Aa}$ & $9,10 \mathrm{Aa}$ & $9,77 \mathrm{Aa}$ & $9,95 \mathrm{Aa}$ & $23,38 \mathrm{Aa}$ & $25,31 \mathrm{Aa}$ & $24,23 \mathrm{Aa}$ & $23,22 \mathrm{Aa}$ \\
\hline $\begin{array}{l}\text { Isolinha parental } \\
\text { (Embrapa 48) }\end{array}$ & \multicolumn{4}{|c|}{9,43} & \multicolumn{4}{|c|}{24,31} \\
\hline $\mathrm{CV}(\%)$ & \multicolumn{4}{|c|}{10,74} & \multicolumn{4}{|c|}{7,71} \\
\hline
\end{tabular}

Médias seguidas pela mesma letra maiúscula na coluna e minúscula na linha não apresentam diferenças estatísticas significativas pelo teste de Scott-Knott a $5 \%$ de probabilidade.

Tabela 4 - Taxa fotossintética e SPAD no estádio V7, após aplicação dos tratamentos com glyphosate e aminoácidos nas plantas de soja (experimento 2)

\begin{tabular}{|c|c|c|c|c|c|c|c|c|}
\hline \multirow{2}{*}{$\begin{array}{l}\text { Glyphosate } \\
\left(\mathrm{g} \text { a.e. } \mathrm{ha}^{-1}\right)\end{array}$} & \multicolumn{4}{|c|}{ Taxa fotossintética (A) $\left(\mathrm{mol} \mathrm{CO}_{2} \mathrm{~m}^{-2} \mathrm{~s}^{-1}\right)$} & \multicolumn{4}{|c|}{ SPAD (unidade) } \\
\hline & SEM AA & TRAT SEM & \begin{tabular}{|c|}
$\begin{array}{c}\text { TRATSEM + } \\
\text { FOL }\end{array}$ \\
\end{tabular} & FOL & SEM AA & TRAT SEM & \begin{tabular}{|c|}
$\begin{array}{c}\text { TRATSEM + } \\
\text { FOL }\end{array}$ \\
\end{tabular} & FOL \\
\hline 0 & $11,02 \mathrm{Aa}$ & $11,01 \mathrm{Aa}$ & $12,50 \mathrm{Aa}$ & $12,23 \mathrm{Aa}$ & $27,03 \mathrm{Aa}$ & ${ }^{(-)} 27,87 \mathrm{Aa}$ & $29,35 \mathrm{Aa}$ & $29,58 \mathrm{Aa}$ \\
\hline 1.200 & ${ }^{(-)} 5,53 \mathrm{Ba}$ & ${ }^{(-)} 7,21 \mathrm{Ba}$ & 9,97 Aa & $9,72 \mathrm{Aa}$ & ${ }^{(-)} 22,67 \mathrm{Aa}$ & ${ }^{(-)} 20,70 \mathrm{Ba}$ & ${ }^{(-)} 22,02 \mathrm{Aa}$ & ${ }^{(-)} 19,43 \mathrm{Ba}$ \\
\hline 2.400 & ${ }^{(-)} 3,69 \mathrm{Ba}$ & ${ }^{(-)} 3,58 \mathrm{Ba}$ & ${ }^{(-)} 4,50 \mathrm{Ba}$ & ${ }^{(-)} 3,54 \mathrm{Ba}$ & ${ }^{(-)} 14,98 \mathrm{Ba}$ & ${ }^{(-)} 14,41 \mathrm{Ba}$ & ${ }^{(-)} 16,02 \mathrm{Ba}$ & ${ }^{(-)} 16,72 \mathrm{Ba}$ \\
\hline $\begin{array}{l}\text { Isolinha parental } \\
\text { (Embrapa 48) }\end{array}$ & \multicolumn{4}{|c|}{12,10} & \multicolumn{4}{|c|}{30,57} \\
\hline $\mathrm{CV}(\%)$ & \multicolumn{4}{|c|}{7,87} & \multicolumn{4}{|c|}{27,28} \\
\hline
\end{tabular}

Médias seguidas pela mesma letra maiúscula na coluna e minúscula na linha não apresentam diferenças significativas pelo teste de ScottKnott a $\mathrm{p} \leq 0,05$. (+, ou -) são comparações pelo teste de Dunnet a $\mathrm{p} \leq 0,05$ com a isolinha parental. 
para acúmulo de biomassa das raízes, em que o tratamento TRATSEM + FOL foi aquele com maior produção de biomassa seca de raiz (Tabela 6). A prevenção da inibição do crescimento de $B$. japonicum tem sido descrita por outros autores, por meio do uso de aminoácidos aromáticos em meios de cultura (Jaworski, 1972; Santos et al., 2005). Supõe-se, portanto, que a fonte exógena aminoácido, cuja composição inclui aminoácidos aromáticos, tenha sido eficaz no suprimento desses aminoácidos eventualmente depletados para os microrganismos simbiontes na soja BRS 242 RR na presença do glyphosate.

Kremer \& Means (2009) também observaram que o glyphosate em soja $R R$ provoca desbalanço de ácido indol acético (AIA), o que leva à menor nodulação de raiz pelo B. japonicum. Como o produto comercial possui em sua composição o aminoácido triptofano, que é um precursor do hormônio AIA (Taiz \& Zeiger, 1998), provavelmente este seja um dos responsáveis pelos efeitos positivos do suprimento exógeno de aminoácidos observados neste experimento. Além disso, a entrada de carbono no sistema-planta, por meio da aplicação de aminoácidos oriundos da fonte exógena, provavelmente repercutiu na prevenção dos efeitos causados pelo glyphosate, com consequente aumento de A (Tabela 5) e da conversão de fotoassimilados (Tabelas 6 e 7).

Há evidências de que o fornecimento, principalmente, de fontes de nitrogênio junto com a aplicação de glyphosate aumentam a injúria das plantas ao herbicida. Reddy \& Zablotowicz (2003), por exemplo, avaliaram diferentes sais de glyphosate, como disopropylamine, trimethylsulfonium (Tms) e diammonium aminomethanamide dihydrogen tetraoxosulfate (Adt), e concluíram que o

Tabela 5 - Taxa fotossintética e SPAD no estádio R1, após aplicação dos tratamentos com glyphosate e aminoácidos nas plantas de soja (experimento 2)

\begin{tabular}{|c|c|c|c|c|c|c|c|c|}
\hline \multirow{2}{*}{$\begin{array}{l}\text { Glyphosate } \\
\left(\text { g a.e. }^{-1} a^{-1}\right)\end{array}$} & \multicolumn{4}{|c|}{ Taxa fotossintética (A) $\left(\mu \mathrm{mol} \mathrm{CO} \mathrm{Cm}^{-2} \mathrm{~s}^{-1}\right)$} & \multicolumn{4}{|c|}{ SPAD (unidade) } \\
\hline & SEM AA & TRAT SEM & $\begin{array}{c}\text { TRATSEM + } \\
\text { FOL }\end{array}$ & FOL & SEM AA & TRAT SEM & $\begin{array}{l}\text { TRATSEM + } \\
\text { FOL }\end{array}$ & FOL \\
\hline 0 & $9,52 \mathrm{Aa}$ & $11,58 \mathrm{Aa}$ & $10,36 \mathrm{Aa}$ & $10,76 \mathrm{Aa}$ & $28,02 \mathrm{Aa}$ & ${ }^{(-)} 24,76 \mathrm{Aa}$ & $27,70 \mathrm{Aa}$ & $27,26 \mathrm{Aa}$ \\
\hline 1.200 & $8,29 \mathrm{Ab}$ & $10,13 \mathrm{Aa}$ & $9,83 \mathrm{Aa}$ & $9,42 \mathrm{Aa}$ & ${ }^{(-)} 23,10 \mathrm{Aa}$ & ${ }^{(-)} 25,21 \mathrm{Aa}$ & ${ }^{(-)} 22,60 \mathrm{Ba}$ & ${ }^{(-)} 23,05 \mathrm{Aa}$ \\
\hline 2.400 & ${ }^{(-)} 5,57 \mathrm{Bb}$ & ${ }^{(-)} 6,09 \mathrm{Bb}$ & $9,69 \mathrm{Aa}$ & ${ }^{(-)} 7,76 \mathrm{Ba}$ & ${ }^{(-)} 16,38 \mathrm{Ba}$ & ${ }^{(-)} 17,00 \mathrm{Ba}$ & ${ }^{(-)} 19,93 \mathrm{Ba}$ & ${ }^{(-)} 21,00 \mathrm{Aa}$ \\
\hline $\begin{array}{l}\text { Isolinha parental } \\
\text { (Embrapa 48) }\end{array}$ & \multicolumn{4}{|c|}{11,48} & \multicolumn{4}{|c|}{27,95} \\
\hline $\mathrm{CV}(\%)$ & \multicolumn{4}{|c|}{27,66} & \multicolumn{4}{|c|}{18,68} \\
\hline
\end{tabular}

Médias seguidas pela mesma letra maiúscula na coluna e minúscula na linha não apresentam diferenças significativas pelo teste de ScottKnott a $\mathrm{p} \leq 0,05$. (+, ou -) são comparações pelo teste de Dunnet a $\mathrm{p} \leq 0,05$ com a isolinha parental

Tabela 6 - Biomassa seca da parte aérea, raiz e total no estádio R1, após aplicação dos tratamentos com glyphosate e aminoácidos nas plantas de soja (experimento 2)

\begin{tabular}{|c|c|c|c|c|c|c|c|c|c|c|c|c|}
\hline \multirow{2}{*}{$\begin{array}{l}\text { Glyphosate } \\
\left(\mathrm{g}^{-} \text {a.e. } \mathrm{ha}^{-1}\right)\end{array}$} & \multicolumn{4}{|c|}{ Parte aérea $(\mathrm{g})$} & \multicolumn{4}{|c|}{ Raiz (g) } & \multicolumn{4}{|c|}{ Total (g) } \\
\hline & SEM AA & $\begin{array}{l}\text { TRAT } \\
\text { SEM }\end{array}$ & \begin{tabular}{|c|} 
TRATSEM \\
+ FOL
\end{tabular} & FOL & SEM AA & $\begin{array}{c}\text { TRAT } \\
\text { SEM }\end{array}$ & \begin{tabular}{|c|} 
TRATSEM \\
+FOL
\end{tabular} & FOL & SEM AA & $\begin{array}{l}\text { TRAT } \\
\text { SEM }\end{array}$ & \begin{tabular}{|c|} 
TRATSEM \\
+ FOL
\end{tabular} & FOL \\
\hline 0 & $12,27 \mathrm{Aa}$ & $11,60 \mathrm{Aa}$ & $11,85 \mathrm{Aa}$ & $12,10 \mathrm{Aa}$ & $3,88 \mathrm{Aa}$ & 4,61 Aa & 3,38 Aa & 4,42 Aa & $16,16 \mathrm{Aa}$ & $16,22 \mathrm{Aa}$ & 15,23 Аa & $16,52 \mathrm{Aa}$ \\
\hline 1.200 & ${ }^{(-)} 8,12 \mathrm{Bb}$ & ${ }^{(-)} 9,16 \mathrm{Ab}$ & $11,92 \mathrm{Aa}$ & $10,70 \mathrm{Aa}$ & $2,59 \mathrm{Bb}$ & $3,34 \mathrm{Ba}$ & $3,76 \mathrm{Aa}$ & $2,96 \mathrm{Bb}$ & ${ }^{(-)} 10,72 \mathrm{Bb}$ & $(-) 12,50 \mathrm{Bb}$ & $15,68 \mathrm{Aa}$ & $13,66 \mathrm{Aa}$ \\
\hline 2.400 & ${ }^{(-)} 8,50 \mathrm{Bb}$ & $(-) 9,84 \mathrm{Ab}$ & $11,90 \mathrm{Aa}$ & $11,47 \mathrm{Aa}$ & ${ }^{(-)} 2,30 \mathrm{Bb}$ & $3,03 \mathrm{Ba}$ & $3,28 \mathrm{Aa}$ & $3,02 \mathrm{Ba}$ & ${ }^{(-)} 10,80 \mathrm{Bb}$ & ${ }^{-(-)} 12,87 \mathrm{Bb}$ & $15,18 \mathrm{Aa}$ & $14,49 \mathrm{Aa}$ \\
\hline $\begin{array}{l}\text { Isolinha } \\
\text { parental } \\
\text { (Embrapa 48) }\end{array}$ & \multicolumn{4}{|c|}{11,80} & \multicolumn{4}{|c|}{3,50} & \multicolumn{4}{|c|}{15,31} \\
\hline CV $(\%)$ & \multicolumn{4}{|c|}{18,69} & \multicolumn{4}{|c|}{24,74} & \multicolumn{4}{|c|}{17,65} \\
\hline
\end{tabular}

Médias seguidas pela mesma letra maiúscula na coluna e minúscula na linha não apresentam diferenças significativas pelo teste de ScottKnott a $\mathrm{p} \leq 0,05$. (+, ou -) são comparações pelo teste de Dunnet a $\mathrm{p} \leq 0,05$ com a isolinha parental 
Tabela 7 - Altura, massa seca de nódulos e número de nódulos no estádio R1, após aplicação dos tratamentos com glyphosate e aminoácidos nas plantas de soja (experimento 2)

\begin{tabular}{|c|c|c|c|c|c|c|c|c|c|c|c|c|}
\hline \multirow{2}{*}{$\begin{array}{l}\text { Glyphosate } \\
\left(\text { g a.e. ha }{ }^{-1}\right)\end{array}$} & \multicolumn{4}{|c|}{ Altura $(\mathrm{cm})$} & \multicolumn{4}{|c|}{ Massa seca de nódulos (mg por planta) } & \multicolumn{4}{|c|}{ Número de nódulos (unidade por planta) } \\
\hline & SEM AA & $\begin{array}{c}\text { TRAT } \\
\text { SEM }\end{array}$ & $\begin{array}{c}\text { TRATSEM } \\
+ \text { FOL }\end{array}$ & FOL & SEM AA & $\begin{array}{c}\text { TRAT } \\
\text { SEM } \\
\end{array}$ & $\begin{array}{c}\text { TRATSEM } \\
+ \text { FOL }\end{array}$ & FOL & SEM AA & $\begin{array}{c}\text { TRAT } \\
\text { SEM }\end{array}$ & $\begin{array}{c}\text { TRATSEM } \\
+ \text { FOL }\end{array}$ & FOL \\
\hline 0 & $16,16 \mathrm{Aa}$ & $16,22 \mathrm{Aa}$ & $15,23 \mathrm{Aa}$ & $16,52 \mathrm{Aa}$ & $572,00 \mathrm{Aa}$ & $395,00 \mathrm{Aa}$ & $387,50 \mathrm{Aa}$ & $452,50 \mathrm{Aa}$ & $115,00 \mathrm{Aa}$ & $85,50 \mathrm{Aa}$ & $98,50 \mathrm{Aa}$ & $93,75 \mathrm{Aa}$ \\
\hline 1.200 & ${ }^{(-)} 10,72 \mathrm{Bb}$ & ${ }^{(-)} 12,50 \mathrm{Bb}$ & $15,68 \mathrm{Aa}$ & $13,66 \mathrm{Aa}$ & $257,00 \mathrm{Bb}$ & $232,50 \mathrm{Bb}$ & $432,50 \mathrm{Aa}$ & $265,00 \mathrm{Ab}$ & $61,50 \mathrm{Ba}$ & $61,50 \mathrm{Aa}$ & $85,50 \mathrm{Aa}$ & $61,75 \mathrm{Aa}$ \\
\hline 2.400 & ${ }^{(-)} 10,80 \mathrm{Bb}$ & ${ }^{(-)} 12,87 \mathrm{Bb}$ & $15,18 \mathrm{Aa}$ & $14,49 \mathrm{Aa}$ & ${ }^{(-)} 180,00 \mathrm{Bb}$ & $257,50 \mathrm{Bb}$ & $392,50 \mathrm{Aa}$ & $292,50 \mathrm{Ab}$ & $(-) 38,00 \mathrm{Bb}$ & $60,75 \mathrm{Ab}$ & $74,75 \mathrm{Aa}$ & $70,50 \mathrm{Aa}$ \\
\hline $\begin{array}{l}\text { Isolinha } \\
\text { parental } \\
\text { (Embrapa 48) }\end{array}$ & \multicolumn{4}{|c|}{15,31} & \multicolumn{4}{|c|}{390,00} & \multicolumn{4}{|c|}{91,25} \\
\hline $\mathrm{CV}(\%)$ & \multicolumn{4}{|c|}{17,65} & \multicolumn{4}{|c|}{17,65} & \multicolumn{4}{|c|}{17,65} \\
\hline
\end{tabular}

Médias seguidas pela mesma letra maiúscula na coluna e minúscula na linha não apresentam diferenças significativas pelo teste de ScottKnott a $\mathrm{p} \leq 0,05$. (+, ou -) são comparações pelo teste de Dunnet a $\mathrm{p} \leq 0,05$ com a isolinha parental.

glyphosate-Adt causou maior injúria na soja $\mathrm{RR}$, apresentando necrose com poucas horas após aplicação, além de redução do número de nódulos, leghemoglobina, peso fresco e clorofila. Bernards et al. (2005) e Mueller et al. (2006) também observaram que a adição de sulfato de amônio aumentou a porcentagem de absorção e translocação de glyphosate, com consequente aumento de efeito no controle de plantas daninhas. Em relação ao efeito da aplicação de potássio junto com glyphosate, não há até o momento evidências de que possa haver antagonismo ou sinergismo da ação do herbicida. Portanto, provavelmente a reversão dos sintomas de intoxicação na soja RR decorre do suprimento exógeno de aminoácidos, e não do suprimento extra de $\mathrm{N}$ e $\mathrm{P}$.

Em resumo, o glyphosate diminuiu a fotossintese e conversão de fotoassimilados nas plantas RR, porém o uso de aminoácidos pode ser uma estratégia para prevenir os efeitos indesejáveis desse herbicida na cultura da soja RR.

\section{AGRADECIMENTOS}

Ao Conselho Nacional de Desenvolvimento Científico e Tecnológico (CNPq), pela bolsa de estudos e pelo apoio financeiro. As marcas comerciais no texto são apenas informativas e não representam nenhuma recomendação oficial do USDA-ARS.

\section{LITERATURA CITADA}

BEALE, S. I. $\delta$-Aminolevulinic acid in plants: its biosynthesis, regulation and role in plastid development. An. Rev. Plant Physiol., v. 29, n. 1, p. 95-120, 1978.
BELLALLOUI, N. et al. Simulated glyphosate drift influences nitrate assimilation and nitrogen fixation in non-glyphosateresistant soybean. J. Agric. Food Chem., v. 54, n. 20, p. 3357-3364, 2006.

BERNARDS, M. L. et al. Glyphosate interaction with manganese in tank mixtures and its effects on glyphosate absorption and translocation. Weed Sci., v. 53, p. 787-794, 2005.

BOTT, S. et al. Glyphosate-induced impairment of plant growth and micronutrient status in glyphosate-resistant soybean (Glycine max L.). Plant Soil, v. 312, n. 1,2, p. 185-194, 2008.

CAKMAK, I. Glyphosate reduced seed and leaf concentrations of calcium, manganese, magnesium and iron in non-glyphosate resistant soybean. Europ. J. Agron., v. 31, n. 1, p. 114-119, 2009

CHEN, S. K.; SUBLER, S.; EDWARDS, C. A. Effects of agricultural biostimulants on soil microbial activity and nitrogen dynamics. Appl. Soil Ecol., v. 19, p. 249-259, 2002.

DE MARIA, N. et al. New insights on glyphosate mode of action in nodulant metabolism: role of shikimate accumulation. J. Agric. Food Chem., v. 54, n. 7, p. 2621-2628, 2006.

DUKE, S. O. et al. Isoflavone, glyphosate, and aminomethylphosphonic acid levels in seeds of glyphosatetreated, glyphosate-resistant soybean. J. Agric. Food Chem., v. 51, n. 1, p. 340-344, 2003.

ECKERT, D. J. Soil test interpretations: Basic cation saturation ratios and sufficiency levels. In: BROWN, J. R. Soil testing: Sampling, correlation, calibration, and interpretation. Madison: SSSA, 1987. p. 53-64. (SSSA Spec. Publ., 21)

EMPRESA BRASILEIRA DE PESQUISA AGROPECUÁRIA - EMBRAPA. Centro Nacional de Pesquisa de Solos Manual de métodos de análises do solo. 2.ed. Rio de Janeiro: 1997. 212 p.

Planta Daninha, Viçosa-MG, v. 28, n. 3, p. 643-653, 2010 
FERREIRA, D. F. Sistema de análise de variância (Sisvar). versão 4.6. Lavras: Universidade Federal de Lavras, 1999. CD ROM.

FORLANI, G. Mode of action of herbicidal derivatives of aminomethylenebisphosphonic acid. Part II. Reversal of herbicidal action by aromatic amino acids. J. Plant Growth Reg., v. 16, n. 1, p. 147-152, 1997.

GRESSHOFF, P. M. Growth inhibition by glyphosate and reversal of its action by phenylalanine and tyrosine. Austr. J. Plant Physiol., v. 6, p. 177-85, 1979

HADERLIE, L. C.; WIDHOLM, J. M.; SLIFE, F. W. Effect of glyphosate on carrot and tobacco cells. Plant Physiol., v. 60, n. 1, p. $40-43,1977$.

HUBER, D. M. Strategies to ameliorate glyphosate immobilization of manganese and its impact on the rhizosphere and disease. In: LORENZ, N.; DICK, R Proceedings of the Glyphosate Potassium Symposium 2006. DeWitt: Ohio State University, AG Spectrum, 2006

JAWORSKI, E. G. Mode of action of N-phosphonomethylglycine: inhibition of aromatic amino acid biosynthesis. $\mathbf{J}$. Agri. Food Chem., v. 20, n. 6, p. 1195-1198, 1972.

JOHAL, G. S.; HUBER, D. M. Glyphosate effects on disease and disease resistance in plants. Europ. J. Agron., v. 31, p. 144-152, 2009.

KABACHNIK, M. I. et al. Organophosphorus complexones. Russian Chem. Rev., v. 43, p. 733-744, 1974.

KING, A. C.; PURCELL, L. C.; VORIES, E. D. Plant growth and nitrogenase activity of glyphosate-tolerant soybean in response to glyphosate applications. Agron. J., v. 93, n. 1, p. 179-186, 2001.

KISHORE, G. M.; SHAH, D. M. Amino acid biosynthesis inhibitors as herbicides. Ann. Rev. Biochem., v. 57, p. 627-663, 1988.

KREMER, R. J.; MEANS, N. E. Glyphosate and glyphosate-resistant crop interactions with rhizosphere microorganisms. Europ. J. Agron., v. 31, n. 1, p. 153-161, 2009

LEE, T. T. Effects of glyphosate on synthesis and degradation of chlorophyll in soybean and tobacco cells. Weed Res., v. 21, n. 1, p. $161-164,1981$.

LIU, F. et al. Stomatal control and water use efficiency of soybean (Glycine max L. Merr.) during progressive soil drying. Environ. Exper. Bot., v. 54, n. 1, p. 33-40, 2005
MOORMAN, T. B. et al. Production of hydroxybenzoic acids by Bradyrhizobium japonicum strains after treatment with glyphosate. J. Agric. Food. Chem., v. 40, n. 1, p. 289293, 1992.

MUELLER, T. C. et al. Comparison of glyphosate salts (isopropylamine, diammonium and potassium) calcium magnesium concentrations on the control various weeds. Weed Technol., v. 20, n. 1, p. 164-171, 2006.

OLIVEIRA JR., R. S. et al. Influência do glyphosate sobre a nodulação e o crescimento de vinte cultivares de soja RR Planta Daninha, v. 26, n. 4, p. 831-843, 2008.

PLINE, W. A.; WU, J.; HATZIOS, K. K. Effects of temperature and chemical additives on the response of transgenic herbicide-resistant soybeans to glufosinate and glyphosate applications. Pestic. Biochem. Physiol., v. 65, n. 1, p. 119-131, 1999.

PROCÓPIO, S. O et al. Características fisiológicas das culturas de soja e feijão e de três espécies de plantas daninhas. Planta Daninha, v. 22, n. 2, p. 211-216, 2004.

RAY, T. B. Inhibition of valine and isoleucine biosynthesis in plants. Plant Physiol., v. 75, p. 827-831, 1984

REDDY, K. N.; HOAGLAND, R. E.; ZABLOTOWICZ, R. $\mathrm{M}$. Effect of glyphosate on growth, chlorophyll content and nodulation in glyphosate-resistant soybeans (Glycine max) varieties. J. New Seeds., v. 2, n. 1, p. 37-52, 2000.

REDDY, K. N.; ZABLOTOWICZ, R. M. Glyphosateresistant soybean response to various salts of glyphosate and glyphosate accumulation in soybean nodules. Weed Sci., v. 51, p. $496-502,2003$

REDDY, K. N.; RIMANDO, A. M.; DUKE, S. O. Aminomethylphosphonic acid, a metabolite of glyphosate, causes injury in glyphosate-treated, glyphosate-resistant soybean. J. Agric. Food Chem., v. 52, n. 16, p. 5139-5143, 2004.

ROISCH, H. U.; LINGENS, F. Angewadte chemie. International Edition 13:400 p. 1974.

SANTOS, J. B. et al. Tolerance of Bradyrhizobium strains to glyphosate formulations. Crop Protec., v. 24, p. 543-547, 2005.

SANTOS, J. B. et al. Efeito de formulações na absorção e translocação do glyphosate em soja transgênica.

Planta Daninha, v. 25, n. 2, p. 381-388, 2007.

SAS Institute. SAS/STAT User Guide. Version 8.2. Cary: 2001. 
SHANER, D. L. et al. Physiological responses of corn to Arsenal. Proc. South Weed Sci., v. 37, p. 364, 1984.

SOUZA, R. A. et al. Avaliação qualitativa e quantitativa da microbiota do solo e da fixação biológica do nitrogênio pela soja. Pesq. Agropec. Bras., v. 43, n. 1, p. 71-82, 2008 a.

SOUZA, R. A. Conjunto mínimo de parâmetros para avaliação da microbiota do solo e da fixação biológica do nitrogênio pela soja. Pesq. Agropec. Bras., v. 43, n. 1, p. 83-91. 2008 b.

SUBLER, S.; DOMINGUEZ, J.; EDWARDS, C.A. Assessing biological activity of agricultural biostimulants: bioassays for plant growth regulators in three soil additives.

Comm. Soil Sci. Plant Anal., v. 29, p. 859-866, 1998.

TAIZ, L.; ZEIGER, E. Plant physiology, Sunderland: Sinauer Associates, 1998. 719 p.
ZABLOTOWICZ, R. M.; REDDY, K. N. Impact of glyphosate on the Bradyrhizobium japonicum symbiosis with glyphosate-resistant transgenic soybean. J. Environ. Qual., v. 33 , n. 3 , p. $825-831,2004$

ZABLOTOWICZ, R. M.; REDDY, K. N. Nitrogenase activity, nitrogen content, and yield responses to glyphosate in glyphosate-resistant soybean. Crop Protec., v. 26, p. $370-376,2007$.

ZOBIOLE, L. H. S. et al. Glyphosate reduces shoot concentration of mineral nutrients in glyphosate-resistant soybeans. Plant Soil, v. 328, n. 1, p. 57-69, 2010a

ZOBIOLE, L. H. S. et al. Effect of glyphosate on symbiotic N2 fixation and nickel concentration in glyphosateresistant soybeans. Applied Soil Ecol., v. 44, p. 176-180, 2010b. 